\title{
PRIOR KNOWLEDGE-BASED DIRECTION OF ARRIVAL ESTIMATION
}

\author{
Petter Wirfält ${ }^{1}$, Magnus Jansson ${ }^{1}$, Guillaume Bouleux ${ }^{2}$, Petre Stoica ${ }^{3}$ \\ ${ }^{1}$ ACCESS Linnaeus Center, Electrical Engineering/Signal Processing lab \\ KTH - Royal Institute of Technology, Sweden \\ ${ }^{2}$ University of Lyon, University of Saint Etienne, LASPI, France \\ ${ }^{3}$ Systems and Control Division, Uppsala University, Uppsala, Sweden
}

\begin{abstract}
In a number of direction of arrival (DOA) estimation applications there exists prior knowledge about the sources whose bearings are to be determined. We study the case when this prior information concerns some of the source positions and their correlation state, which is a relevant case in, for example, RADAR scenarios where stationary objects exists in the regions of interest. Traditional DOA methods are not designed to exploit such information, and thus cannot obtain the highest theoretical accuracy. We present a method that can utilize in an asymptotically efficient manner both knowledge on some source positions and that the source signals are uncorrelated.
\end{abstract}

Index Terms - Antenna arrays, Array signal processing, Direction of arrival estimation, Sensor arrays

\section{INTRODUCTION}

Direction of arrival (DOA) estimation is a much studied topic in signal processing, and based on a given scenario there is usually one particular algorithm that is desirable from a theoretical point of view. When studying e.g. uniform linear arrays (ULAs), a very effective algorithm has been shown to be MODE [1]. This algorithm is asymptotically statistically efficient in most scenarios. However, it cannot efficiently incorporate the knowledge that the sources are uncorrelated, should this be the case. A method that successfully does this is presented in [2]. Another highly popular method is MUSIC [3], but this algorithm is in general less efficient than MODE. It does however allow for arbitrary array geometries.

None of the above mentioned algorithms can inherently incorporate prior knowledge on some of the present DOAs; the prior information is concealed in the received data, and the algorithms will simply treat the known directions as additional unknowns. The situation of prior bearing knowledge arises in a number of applications. Consider for example a

The research leading to these results has received funding from the European Research Council under the European Community's Seventh Framework Programme (FP7/2007-2013) / ERC grant agreement \#228044.
RADAR scenario, where a given sector is continuously observed. If there is a reflector such as an obstacle within the sector, a reflection will be known to be present whenever the sector is analyzed. It is then desirable to be able to exploit this information rather than to simply blank the appearing target resulting from the object. Further application examples are described in [4].

Previously, some modifications of the MUSIC algorithm have been proposed, in which known directions are cancelled through orthogonal projections [5], [6]. As mentioned, MUSIC does not in general give state-of-the-art performance; thus the utilization of prior information cannot be expected to be entirely satisfactory either. A recent proposal in this area is [7], the novelty of which was to use oblique rather than orthogonal projections.

In this paper we use a method that is known to perform optimally in the sense of asymptotic accuracy. Then, we incorporate the prior knowledge in an optimal manner, such that no information is lost (the previously mentioned projection based approaches are by construction non-bijective, and hence information is lost.) The basic idea of utilizing prior knowledge in this way was first proposed in [8], where it was explored in conjunction with the MODE-algorithm [1].

The contribution of this paper is that we implement the algorithm in a different estimator [2], and that we show the possibility of a dramatic increase in the estimator accuracy when combining the information on the uncorrelation of the signals with prior knowledge on some of the DOAs. We illustrate this significant accuracy gain by a numerical example using simulated data. In the full version of this paper, [4], we perform additional studies using real-data to track moving targets, as well as covariance-based frequency estimation on real data in the presence of strong known signals.

\section{DATA MODEL}

We consider the problem of estimating the DOAs of $d$ narrowband signals impinging on a ULA consisting of $m$ sensors. We assume the signals to be uncorrelated, and also that the number of signals $d$ is known. 
Let the vector of true DOAs be $\boldsymbol{\theta}=\left[\begin{array}{llll}\theta_{1} & \theta_{2} & \cdots & \theta_{d}\end{array}\right]^{T}$, measured from the array broadside, and let the superscript $T$ denote the transpose operation. We also assume that some $\left\{\theta_{i}\right\}$ are known a priori.

We observe $N$ snapshots of the sensor outputs defined according to

$$
\mathbf{y}(t)=\mathbf{A}(\boldsymbol{\theta}) \mathbf{s}(t)+\mathbf{n}(t), \quad t=1, \ldots, N,
$$

where $\mathbf{A}(\boldsymbol{\theta})=\left[\begin{array}{lll}\mathbf{a}\left(\theta_{1}\right) & \cdots & \mathbf{a}\left(\theta_{d}\right)\end{array}\right]$ is the Vandermonde array response where $\mathbf{a}\left(\theta_{i}\right)=\left[\begin{array}{llll}1 & e^{j \varphi_{i}} & \cdots & e^{j(m-1) \varphi_{i}}\end{array}\right]^{T}$ in which

$$
\varphi_{i}=-2 \pi \Delta \sin \left(\theta_{i}\right),
$$

and $\Delta$ is the intra-sensor spacing. In (1), $\mathbf{s}(t)$ and $\mathbf{n}(t)$ are the vectors of source signals and of the signal-independent sensor noise at time $t$, respectively, which both are zero mean, complex-Gaussian, temporally uncorrelated random processes with second-order moments given by

$$
\begin{aligned}
\mathrm{E}\left[\mathbf{s}(t) \mathbf{s}^{*}(t)\right] & =\mathbf{P}=\operatorname{diag}\left(p_{1}, \ldots, p_{d}\right), \\
\mathrm{E}\left[\mathbf{n}(t) \mathbf{n}^{*}(t)\right] & =\sigma^{2} \mathbf{I} .
\end{aligned}
$$

Hereafter, the superscript ${ }^{*}$ denotes the conjugate transpose. Further, let

$$
\mathbf{R}=\mathrm{E}\left[\mathbf{y}(t) \mathbf{y}^{*}(t)\right]
$$

be the data covariance matrix, and define a sample version of it as

$$
\hat{\mathbf{R}}=\frac{1}{N} \sum_{t=1}^{N} \mathbf{y}(t) \mathbf{y}^{*}(t)
$$

\section{DOA ESTIMATION}

The contribution of this paper is to incorporate prior knowledge on some DOAs in the estimation process of the others. To this end, we utilize an existing DOA finding method [2], which is asymptotically statistically efficient under the assumptions in Section 2. In order to describe our approach, some preliminaries are needed.

When the signals impinging on the ULA are uncorrelated, the data covariance matrix (5) is Toeplitz and can be written as

$$
\mathbf{R}=\left[\begin{array}{cccc}
r(0) & r(-1) & \ldots & r(-m+1) \\
r(1) & r(0) & & \\
\vdots & & \ddots & \vdots \\
r(m-1) & r(m-2) & \ldots & r(0)
\end{array}\right]
$$

Following [2], we define $\mathbf{r}=\operatorname{vec}(\mathbf{R})$ and $\hat{\mathbf{r}}=\operatorname{vec}(\hat{\mathbf{R}})$. We have that:

$$
\mathbf{r}=\boldsymbol{\Phi} \rho
$$

with

$$
\boldsymbol{\rho}=\left[\begin{array}{llll}
r(-m+1) & r(-m+2) & \cdots & r(m-1)
\end{array}\right]^{T}
$$

and $\Phi$ a selection matrix with an evident definition. Using the data model in (1), the vector $\rho$ in (8) can be written as

$$
\boldsymbol{\rho}=\overline{\mathbf{A}} \mathbf{p}+\sigma^{2} \mathbf{e}
$$

where $\mathbf{p}=\left[\begin{array}{llll}p_{1} & p_{2} & \cdots & p_{d}\end{array}\right]^{T}$ are the non-zero diagonal elements of the signal covariance matrix (3). In addition, e is a $(2 m-1) \times 1$ vector of zeros except for a one in the $m$ th position and $\overline{\mathbf{A}}$ is the extended array response matrix defined as

$$
\overline{\mathbf{A}}=\left[\begin{array}{ccc}
e^{j(-m+1) \varphi_{1}} & \cdots & e^{j(-m+1) \varphi_{d}} \\
e^{j(-m+2) \varphi_{1}} & \cdots & e^{j(-m+2) \varphi_{d}} \\
\vdots & & \vdots \\
e^{j(m-1) \varphi_{1}} & \cdots & e^{j(m-1) \varphi_{d}}
\end{array}\right]
$$

We can thus find DOA estimates by minimizing the criterion

$$
V(\boldsymbol{\eta})=[\hat{\mathbf{r}}-\mathbf{r}(\boldsymbol{\eta})]^{*} \mathbf{W}[\hat{\mathbf{r}}-\mathbf{r}(\boldsymbol{\eta})],
$$

where $\boldsymbol{\eta}=\left[\begin{array}{lll}\boldsymbol{\theta}^{T} & \mathbf{p}^{T} & \sigma^{2}\end{array}\right]^{T}$ and $\mathbf{W}$ is a suitably chosen weighting matrix. Following [2] and [9], we choose

$$
\mathbf{W}=\hat{\mathbf{R}}^{-T} \otimes \hat{\mathbf{R}}^{-1}
$$

where $\otimes$ denotes the Kronecker product. In order to write (11) in a more convenient form, we introduce the following weighted variables:

$$
\begin{aligned}
\hat{\mathbf{r}}_{\mathbf{w}} & =\mathbf{W}^{1 / 2} \hat{\mathbf{r}}, \quad \boldsymbol{\Phi}_{\mathbf{w}}=\mathbf{W}^{1 / 2} \boldsymbol{\Phi} \\
\overline{\mathbf{A}}_{\mathbf{w}} & =\left(\boldsymbol{\Phi}_{\mathbf{w}}^{*} \boldsymbol{\Phi}_{\mathbf{w}}\right)^{1 / 2} \overline{\mathbf{A}}, \quad \mathbf{e}_{\mathbf{w}}=\left(\boldsymbol{\Phi}_{\mathbf{w}}^{*} \boldsymbol{\Phi}_{\mathbf{w}}\right)^{1 / 2} \mathbf{e}
\end{aligned}
$$

and

$$
\hat{\boldsymbol{\rho}}_{\mathbf{w}}=\left(\boldsymbol{\Phi}_{\mathbf{w}}^{*} \boldsymbol{\Phi}_{\mathbf{w}}\right)^{-1 / 2} \boldsymbol{\Phi}_{\mathbf{w}}^{*} \hat{\mathbf{r}}_{\mathbf{w}}
$$

where $(\cdot)^{1 / 2}$ denotes Hermitian matrix square root. Using this notation, we rewrite (11) as (neglecting a constant term) [2]

$$
V(\boldsymbol{\eta})=\left\|\hat{\boldsymbol{\rho}}_{\mathbf{w}}-\overline{\mathbf{A}}_{\mathbf{w}} \mathbf{p}-\mathbf{e}_{\mathbf{w}} \sigma^{2}\right\|_{2}^{2} .
$$

For fixed $\theta$ and $\sigma^{2}$ we can directly find the $\mathbf{p}$ that minimizes (14) as

$$
\hat{\mathbf{p}}\left(\boldsymbol{\theta}, \sigma^{2}\right)=\overline{\mathbf{A}}_{\mathbf{w}}^{\dagger}\left(\hat{\boldsymbol{\rho}}_{w}-\mathbf{e}_{\mathbf{w}} \sigma^{2}\right),
$$

where $^{\dagger}$ represents the Moore-Penrose pseudoinverse. To proceed, we need to exploit the properties of the Vandermonde matrix $\overline{\mathbf{A}}$. In order to do this, we define the following polynomial, based on the $\varphi_{1}, \ldots, \varphi_{d}$ corresponding to the true DOAs,

$$
b_{0} z^{d}+b_{1} z^{d-1}+\ldots+b_{d}=b_{0} \prod_{i=1}^{d}\left(z-e^{j \varphi_{i}}\right),
$$

whose coefficients are, by definition, conjugate symmetric. Using these polynomial coefficients, we define the matrix

$$
\mathbf{B}^{*}=\left[\begin{array}{ccccccc}
b_{d} & \ldots & b_{1} & b_{0} & 0 & \ldots & 0 \\
0 & b_{d} & \ldots & b_{1} & b_{0} & \ddots & \vdots \\
\vdots & \ddots & \ddots & & \ddots & \ddots & 0 \\
0 & \ldots & 0 & b_{d} & \ldots & b_{1} & b_{0}
\end{array}\right]
$$


of dimension $(2 m-1) \times(2 m-1-d)$. Since $\mathbf{B}^{*} \overline{\mathbf{A}}=0$, $\boldsymbol{\Pi}_{\overline{\mathbf{A}}}^{\perp}=\boldsymbol{\Pi}_{\mathbf{B}}$, where $\boldsymbol{\Pi}_{\mathbf{X}}=\mathbf{X}\left(\mathbf{X}^{*} \mathbf{X}\right)^{-1} \mathbf{X}^{*}$ is the orthogonal projection onto the range space of $\mathbf{X}$, and $\Pi_{\mathbf{X}}^{\perp}=\mathbf{I}-\boldsymbol{\Pi}_{\mathbf{X}}$. Let

$$
\mathbf{B}_{\mathbf{w}}=\left(\boldsymbol{\Phi}_{\mathbf{w}}^{*} \boldsymbol{\Phi}_{\mathbf{w}}\right)^{-1 / 2} \mathbf{B}
$$

and similarly to $\Pi_{\overline{\mathbf{A}}}^{\perp}=\Pi_{\mathbf{B}}$ we have that $\Pi_{\overline{\mathbf{A}}_{\mathbf{w}}}^{\perp}=\boldsymbol{\Pi}_{\mathbf{B}_{\mathrm{w}}}$. From (14) and (15) we can then find an estimate of $\sigma^{2}$ as

$$
\bar{\sigma}^{2}(\boldsymbol{\theta})=\left(\mathbf{e}_{\mathbf{w}}^{*} \boldsymbol{\Pi}_{\mathbf{B}_{\mathbf{w}}} \mathbf{e}_{\mathbf{w}}\right)^{-1} \mathbf{e}_{\mathbf{w}}^{*} \boldsymbol{\Pi}_{\mathbf{B}_{\mathbf{w}}} \hat{\boldsymbol{\rho}}_{\mathbf{w}} .
$$

Using the estimates (15) and (19) we can rewrite (14) as

$$
V(\boldsymbol{\theta})=\left\|\boldsymbol{\Pi}_{\mathbf{B}_{\mathbf{w}}}\left(\hat{\boldsymbol{\rho}}_{\mathbf{w}}-\mathbf{e}_{\mathbf{w}} \bar{\sigma}^{2}\right)\right\|_{2}^{2} .
$$

It can be shown (see [2]) that (20) is asymptotically equivalent to

$$
V(\mathbf{b})=\left(\hat{\boldsymbol{\rho}}_{\mathbf{w}}-\mathbf{e}_{\mathbf{w}} \hat{\sigma}^{2}\right)^{*} \mathbf{B}_{\mathbf{w}} \hat{\boldsymbol{\Sigma}} \mathbf{B}_{\mathbf{w}}^{*}\left(\hat{\boldsymbol{\rho}}_{\mathbf{w}}-\mathbf{e}_{\mathbf{w}} \hat{\sigma}^{2}\right),
$$

where $\mathbf{b}=\left[\begin{array}{lll}b_{0} & \ldots & b_{d}\end{array}\right]^{T}, \hat{\mathbf{\Sigma}}$ is a consistent estimate of

$$
\boldsymbol{\Sigma}=\mathbf{B}_{\mathbf{w}}^{\dagger} \boldsymbol{\Pi}_{\boldsymbol{\Pi}_{\mathbf{B}_{\mathbf{w}} \mathbf{e}_{\mathbf{w}}}^{\perp}}\left(\mathbf{B}_{\mathbf{w}}^{\dagger}\right)^{*},
$$

and $\hat{\sigma}^{2}$ is a consistent estimate of $\sigma^{2}$. The somewhat involved expression (22) follows from the use of quantities estimated from data in (21) and the need for asymptotic equivalency between (20) and (21). If we introduce the matrix

$$
\mathbf{Q}=\left[\begin{array}{ccc}
q_{d+1} & \cdots & q_{1} \\
\vdots & & \vdots \\
q_{2 m-1} & \cdots & q_{2 m-d-1}
\end{array}\right]
$$

in which

$$
\left[\begin{array}{lll}
q_{1} & \cdots & q_{2 m-1}
\end{array}\right]^{T} \triangleq\left(\boldsymbol{\Phi}_{\mathbf{w}}^{*} \boldsymbol{\Phi}_{\mathbf{w}}\right)^{-1 / 2}\left(\hat{\boldsymbol{\rho}}_{\mathbf{w}}-\mathbf{e}_{\mathbf{w}} \hat{\sigma}^{2}\right),
$$

we can rewrite (21) as

$$
\begin{aligned}
V(\mathbf{b}) & =\left(\hat{\boldsymbol{\rho}}_{\mathbf{w}}-\mathbf{e}_{\mathbf{w}} \hat{\sigma}^{2}\right)^{*} \mathbf{B}_{\mathbf{w}} \hat{\boldsymbol{\Sigma}} \mathbf{B}_{\mathbf{w}}^{*}\left(\hat{\boldsymbol{\rho}}_{\mathbf{w}}-\mathbf{e}_{\mathbf{w}} \hat{\sigma}^{2}\right) \\
& =\mathbf{b}^{*} \mathbf{Q}^{*} \hat{\boldsymbol{\Sigma}} \mathbf{Q b} .
\end{aligned}
$$

In this way we obtain the criterion function in a form suitable for minimization with respect to b, e.g., by the eigendecomposition of $\mathbf{Q}^{*} \hat{\boldsymbol{\Sigma}} \mathbf{Q}[2]$.

\subsection{Prior bearing knowLEDGE (PLEDGE)}

The method above does not discriminate between known and unknown DOAs. We now show how to incorporate this information into the estimator. Since the true DOAs define the roots of the polynomial (16), knowledge of some DOA values translates into knowledge of some roots of this polynomial. Thus, we can factor the polynomial into two factors: one associated with the known DOAs and one associated with the unknown ones. Consequently, we can retain only the polynomial coefficients associated with the unknown sources as parameters to estimate.

Following the above idea, we write (16) as

$$
b_{0} \prod_{i=1}^{d}\left(z-e^{j \varphi_{i}}\right)=P_{k}(z) P_{u}(z)
$$

in which

$$
P_{u}(z)=\tilde{b}_{0} \prod_{i=1}^{d_{u}}\left(z-e^{j \varphi_{i}}\right)=\tilde{b}_{0} z^{d_{u}}+\ldots+\tilde{b}_{d_{u}}
$$

and

$$
P_{k}(z)=c_{0} \prod_{i=d_{u}+1}^{d}\left(z-e^{j \varphi_{i}}\right)=c_{0} z^{d_{k}}+\ldots+c_{d_{k}} .
$$

Here, $P_{k}(z)$ is the polynomial with $d_{k}$ zeros corresponding to the known DOAs whereas $P_{u}(z)$ has $d_{u}=d-d_{k}$ zeros corresponding to the unknown DOAs. We use (25)-(27) to write

$$
\mathbf{b}=\mathbf{C} \tilde{\mathbf{b}}
$$

where

$$
\tilde{\mathbf{b}}=\left[\begin{array}{llll}
\tilde{b}_{0} & \tilde{b}_{1} & \ldots & \tilde{b}_{d_{u}}
\end{array}\right]^{T}
$$

and

$$
\mathbf{C}^{T}=\left[\begin{array}{cccccc}
c_{0} & c_{1} & \ldots & c_{d_{k}} & & \mathbf{0} \\
& \ddots & \ddots & & \ddots & \\
\mathbf{0} & & c_{0} & c_{1} & \ldots & c_{d_{k}}
\end{array}\right],
$$

with $\mathbf{C} \in \mathbb{C}^{(d+1) \times\left(d_{u}+1\right)}$.

Substituting (28) in (24), we obtain the PLEDGE uncorrelated (PUC) criterion function

$$
V_{\mathrm{PUC}}(\tilde{\mathbf{b}})=\tilde{\mathbf{b}}^{*} \mathbf{C}^{*} \mathbf{Q}^{*} \hat{\mathbf{\Sigma}} \mathbf{Q} \mathbf{C} \tilde{\mathbf{b}} .
$$

The proposed DOA finding algorithm can now be summarized as follows:

1. Find $\hat{\boldsymbol{\rho}}_{\mathrm{w}}$ from $\hat{\mathbf{R}}$ using (13); form $\mathbf{C}$ in (30) from the known DOAs.

2. Obtain an initial estimate of $\hat{\sigma}^{2}$, e.g. by averaging the $m-d$ smallest eigenvalues of $\hat{\mathbf{R}}$. Initialize $\hat{\boldsymbol{\Sigma}}=\mathbf{I}$, and form $\mathbf{Q}$ using (23).

3. Find an estimate of $\tilde{\mathbf{b}}$ by minimizing (31) subject to $\|\mathbf{b}\|_{2}=1$; then find an initial estimate of $\mathbf{b}$ from (28); use this estimate in (18) to find $\mathbf{B}_{\mathbf{w}}$. Then use (19) to find a more accurate $\hat{\sigma}^{2}$, and use (22) to find the weighting matrix $\hat{\boldsymbol{\Sigma}}$.

4. Using the above enhanced estimates, minimize (31) once again to find $\tilde{\mathbf{b}}$, then obtain $\varphi_{i}, i=1, \ldots, d_{u}$, by rooting (26), and finally the sought DOAs through (2). 


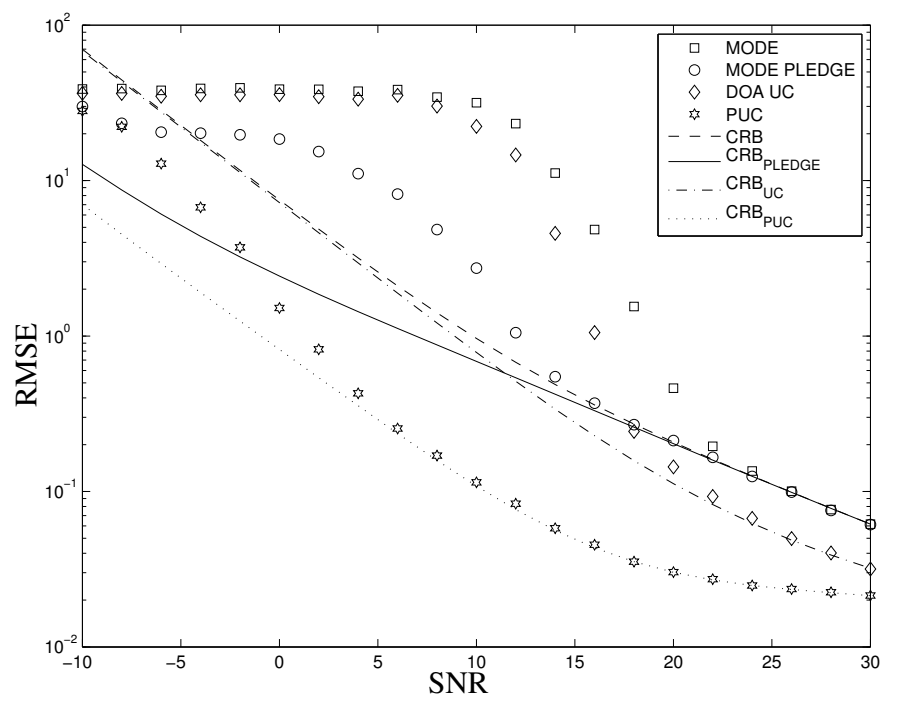

Fig. 1. RMSE-values (in degrees) for four estimators of $\theta_{1}=$ $10^{\circ}$, and their respective theoretical accuracy bounds; $\boldsymbol{\theta}=$ $\left[\begin{array}{lll}10^{\circ} & 12^{\circ} & 14^{\circ}\end{array}\right]^{T}$, with $\theta_{2}=12^{\circ}$ considered known. $N=$ $1000, m=10$, and $1000 \mathrm{MC}$ realizations. The sources are uncorrelated and equipowered.

\section{SIMULATIONS}

In order to evaluate the proposed approach we perform Monte Carlo (MC) simulations in which we compare PUC to the unmodified estimator presented in [2]. In addition, we also include the estimates given by MODE [1] and its PLEDGE derivative [8]. The latter two estimators are statistically efficient only if the uncorrelation of the sources is not taken into account, as they cannot exploit the information that the signals are uncorrelated.

We use $m=10$ and generate $N=1000$ simulated snapshots in each simulation realization in order to form the sample covariance matrix $\hat{\mathbf{R}}$. We compare the DOA Root Mean Square Error (RMSE) of each estimator, and we let $\mathrm{SNR}_{i}=$ $p_{i} / \sigma^{2}$ denote the signal to noise ratio of signal $i$.

In the simulated scenario we consider three equipowered sources at $\boldsymbol{\theta}=\left[\begin{array}{lll}10^{\circ} & 12^{\circ} & 14^{\circ}\end{array}\right]^{T}$ and with $\theta_{2}=12^{\circ}$ known. The performance of each considered estimator can be seen in Fig. 1. In the figure we also show several Cramér Rao lower Bounds (CRBs) which each correspond to different assumptions on the available knowledge. The CRB without subscript refers to an estimator with no prior knowledge [10], PLEDGE refers to prior bearing knowledge [8], UC refers to prior knowledge regarding the uncorrelation of sources [11], and PUC refers to the bound when using both uncorrelation and bearing prior knowledge [4].

We notice that the devised estimator approaches its theoretical bound at lower SNRs than the other algorithms do, and that this bound is significantly lower than the other estimators' bounds. This means that we can correctly resolve sources at significantly lower SNRs than previously possible. We see the benefit of using prior information for PLEDGE MODE as well, but this benefit vanishes earlier as SNR increases than the benefit gained by the UC-estimators. It can also be seen that the benefit of prior DOA knowledge decreases as SNR increases. We omit the results pertaining to the estimation of $\theta_{3}=14^{\circ}$ as they are very similar to those shown in Fig. 1 .

\section{CONCLUSIONS}

We have shown how to incorporate prior information on source bearings in a scenario where the sources are also known to be uncorrelated. Our results imply that being able to exploit the prior information regarding both source bearings and source decorrelation can provide significant accuracy increases.

\section{REFERENCES}

[1] P. Stoica and K. Sharman, "Maximum likelihood methods for direction-of-arrival estimation," IEEE Trans. Acoust., Speech, Signal Process., vol. 38, no. 7, pp. 1132-1143, Jul. 1990.

[2] M. Jansson and B. Ottersten, "Structured covariance matrix estimation: A parametric approach," in IEEE Int. Conf. on Acoustics, Speech and Signal Processing, Istanbul, Turkey, Jun. 2000, pp. 3172-75.

[3] R.O. Schmidt, "Multiple emitter location and signal parameter estimation," in Proc. of RADC Spectrum Estimation Workshop, 1979, pp. 243-258.

[4] P. Wirfält, G. Bouleux, M. Jansson, and P. Stoica, "Optimal prior knowledge-based direction of arrival estimation," In preparation, Oct. 2010.

[5] R.D. DeGroat, E.M. Dowling, and D.A. Linebarger, "The constrained MUSIC problem," IEEE Trans. Signal Process., vol. 41, no. 3, pp. 1445-1449, 1993.

[6] D.A. Linebarger, R.D. DeGroat, E.M. Dowling, P. Stoica, and G.L. Fudge, "Incorporating a priori information into MUSICalgorithms and analysis," Signal Process., vol. 46, no. 1, pp. 85-104, 1995.

[7] R. Boyer and G. Bouleux, "Oblique projections for directionof-arrival estimation with prior knowledge," IEEE Trans. Signal Process., vol. 56, no. 4, pp. 1374-1387, Apr. 2008.

[8] G. Bouleux, P. Stoica, and R. Boyer, "An optimal prior knowledge-based DOA estimation method," in 17th European Signal Processing Conference, Glasgow, UK, Aug. 2009, pp. 869-873.

[9] H. Li, P. Stoica, and J. Li, "Computationally efficient maximum likelihood estimation of structured covariance matrices," IEEE Trans. Signal Process., vol. 47, no. 5, pp. 1314-23, May 1999.

[10] P. Stoica, E.G. Larsson, and A.B. Gershman, "The stochastic CRB for array processing: a textbook derivation," IEEE Signal Processing Letters, vol. 8, no. 5, pp. 148-150, 2001.

[11] M. Jansson, B. Göransson, and B. Ottersten, "A subspace method for direction of arrival estimation of uncorrelated emitter signals," IEEE Trans. Signal Process., vol. 47, no. 4, pp. 945-956, Apr. 1999. 